\title{
Induction of Activating Transcription Factors (ATFs) ATF2, ATF3, and ATF4 in the Nucleus Accumbens and Their Regulation of Emotional Behavior
}

\author{
Thomas A. Green, ${ }^{1}$ Imran N. Alibhai, ${ }^{1}$ Stephen Unterberg, ${ }^{1}$ Rachael L. Neve, ${ }^{2}$ Subroto Ghose, ${ }^{1}$ Carol A. Tamminga, ${ }^{1}$ and \\ Eric J. Nestler ${ }^{1}$ \\ ${ }^{1}$ Departments of Psychiatry and Neuroscience, The University of Texas Southwestern Medical Center, Dallas, Texas 75390-9070, and ${ }^{2}$ McLean Hospital, \\ Department of Psychiatry, Harvard University School of Medicine, Belmont, Massachusetts 02478
}

\begin{abstract}
Previous research has shown that cAMP response element (CRE) binding protein (CREB) in the nucleus accumbens gates behavioral responses to emotional stimuli. For example, overexpression of CREB decreases anxiety, sucrose preference, and sensitivity to drugs of abuse and increases depression-like behavior, whereas blocking CREB via overexpression of inducible cAMP early repressor (ICER) or other dominant-negative inhibitors of CRE-mediated transcription has the opposite effects. However, CREB and ICER are but two members of a larger family of leucine zipper-containing transcription factors composed of multiple products of the creb, crem (cAMP response element modulator), and atf (activating transcription factor) genes. We demonstrate here that ATF2, ATF3, and ATF4 are each robustly induced in the nucleus accumbens and dorsal striatum by restraint stress or by amphetamine administration. In contrast, little induction is seen for ATF1 or CREM. Using viral-mediated gene transfer, we show that ATF2 overexpression in nucleus accumbens produces increases in emotional reactivity and antidepressant-like responses, a behavioral phenotype similar to that caused by dominant-negative antagonists of CREB. In contrast, ATF3 or ATF4 overexpression in nucleus accumbens decreases emotional reactivity and increases depression-like behavior, consistent with the behavioral phenotype induced by CREB. Because amphetamine and stress induce ATF2, ATF3, and ATF4 in nucleus accumbens, and overexpression of these transcription factors in this brain region in turn alters behavioral responsiveness to amphetamine and stress, our findings support novel roles for these ATF family members in regulating emotional behavior.
\end{abstract}

Key words: elevated plus maze; anhedonia; porsolt; forced swim test; addiction; anxiety; drugs of abuse; stimulants; CREB; CREM; striatum; sucrose preference; neophobia; behavior; CRE-BP1; CREB2

\section{Introduction}

Increasing evidence supports a role for the transcription factor cAMP response element (CRE) binding protein (CREB), acting in the nucleus accumbens (a major reward region of brain), to regulate behavioral responses to emotional stimuli. Overexpression of CREB, via viral-mediated gene transfer or in inducible bitransgenic mice, decreases emotional reactivity as evidenced by decreased anxiety-like behavior, decreased responses to drugs of abuse and natural rewards, and increased depression-like behavior. In contrast, overexpression of inducible cAMP early repressor (ICER), a naturally occurring dominant-negative antagonist of CREB, or synthetic dominant-negative CREB mutants (e.g., mCREB), exert the opposite effects (Carlezon et al., 1998; Barrot et al., 2002; Newton et al., 2002; Sakai et al., 2002; Green et al.,

\footnotetext{
Received Dec. 27, 2006; revised Jan. 9, 2008; accepted Jan. 9, 2008.

This work was supported by grants from the National Institute of Mental Health and the National Institute on Drug Abuse. We thank Ami Graham and Sherri Khatami for technical assistance and John Neumaier (University of Washington) for development of the bicistronic viral vector

Correspondence should be addressed to Eric J. Nestler at the above address. E-mail: eric.nestler@utsouthwestern.edu.

DOI:10.1523/JNEUROSCI.5273-07.2008

Copyright $\odot 2008$ Society for Neuroscience $\quad$ 0270-6474/08/282025-08\$15.00/0
}

2006; Pittenger et al., 2006). Studies of mice deficient in the two major isoforms of CREB generally support these conclusions (Walters and Blendy, 2001; Walters et al., 2003; Blendy, 2006).

However, CREB and ICER are just two members of a much larger family of structurally and functionally related transcription factors, which also include isoforms of cAMP response element modulator (CREM) and several activating transcription factors (ATFs). These CREB/ATF family proteins are categorized as basic region- and leucine zipper-containing (bZIP) transcription factors, which can homodimerize or heterodimerize to regulate gene transcription. Although CREB, CREM, and ATF1 are relatively well characterized and known to regulate gene transcription via binding to CRE sites, ATF2, ATF3, and ATF4 are structurally more distant, and their functional properties, particularly in brain, remain poorly understood. Rather than being activated by the cAMP cascade, ATF2 is activated by c-Jun N-terminal kinase (JNK) and can dimerize with members of the activator protein-1 (AP-1) family such as c-Jun to bind CRE or AP-1 sites (Hai and Curran, 1991; De Cesare et al., 1995). Additionally, ATF2 homodimers and ATF2/c-Jun heterodimers can bind certain CRElike sites that are insensitive to CREB (Benbrook and Jones, 1994). ATF3 and ATF4 also dimerize with various Jun species and 
can shift c-Jun DNA binding site preferences from AP-1 to CRE, thereby promoting cross talk among AP-1 and CREB families of proteins (Hai and Curran, 1991).

ATF2, ATF3, and ATF4 are often studied as cellular stress response proteins (Hai et al., 1999; Wek et al., 2006), but recent evidence suggests that these transcription factors may be involved in nonstress adaptations. This has prompted reclassification of these proteins as more general "adaptive response" transcription factors (Cho et al., 2001; Lu et al., 2007). There is one report of alterations in ATF2 levels in frontal cortex after chronic administration of antidepressants (Laifenfeld et al., 2004). However, virtually nothing is known about the function of these transcription factors in the nucleus accumbens or in response to drugs of abuse or behavioral stress. We therefore set out to study the regulation of ATF2, ATF3, and ATF4 in this brain region after amphetamine treatment or restraint stress and characterized the influence of overexpression of these proteins in the nucleus accumbens on complex emotional behavior.

\section{Materials and Methods}

Animals. Male Sprague Dawley rats (Harlan, Houston, TX), 250-350 g, were used for all experiments. Rats were pair-housed in an Association for Assessment and Accreditation of Laboratory Animal Care-approved colony, and all experiments conformed to the NIH Guide for the Use and Care of Laboratory Animals and the University of Texas Southwestern Institutional Animal Care and Use Committee. Rats were housed on a $12 \mathrm{~h} \mathrm{light/dark} \mathrm{cycle} \mathrm{with} \mathrm{lights} \mathrm{on} \mathrm{at} \mathrm{7:00} \mathrm{A.M.} \mathrm{All} \mathrm{procedures} \mathrm{were}$ conducted during the light phase of the cycle.

Statistical analyses. All data were analyzed using ANOVA methods. Where appropriate, planned comparisons or Bonferroni's post hoc procedures were used. All statistical $p$ values are for a two-tailed test unless noted.

Quantification of $m R N A$. Rats were killed by rapid decapitation, nucleus accumbens and dorsal striatum were dissected, and mRNA was isolated using the RNA Stat-60 reagent (Teltest, Houston, TX) according to manufacturer directions. Contaminating DNA was removed with DNase treatment (DNA-Free, catalog \#1906; Ambion, Austin, TX). Purified RNA was reverse-transcribed into cDNA (Superscript First Strand Synthesis; catalog \#12371-019; Invitrogen). ATF2, ATF3, and ATF4 transcripts were quantified using real-time PCR (SYBR Green; Applied Biosystems, Foster City, CA) on a Stratagene (La Jolla, CA) Mx5000p 96-well thermocycler. Primers for rat ATF2 (forward: CCTCTTGCAACACCCATCA; reverse: TTTGTGCCAATGGTATTTCCT), ATF3 (forward: GCCATCCAGAACAAGCACC; reverse: CACTTGGCAGCAGCAATTT), and ATF4 (forward: CCTTCGACCAGTCGGGTTTG; reverse: CTGTCCCGGAAAAGGCATCC) were validated for linearity and specificity before experiments. All PCR data were normalized to glyceraldehyde3-phosphate dehydrogenase levels, which were not altered by stress or amphetamine treatments.

Amphetamine treatment. D-Amphetamine sulfate (Sigma, Dallas, TX) was administered at a dose of $4 \mathrm{mg} / \mathrm{kg}$ (i.p.). Rats received 7 daily injections of saline or amphetamine. Thus, for example, "acute" amphetamine animals received six daily injections of saline, followed by an amphetamine injection on day 7 , whereas " $7 \mathrm{~d}$ " amphetamine animals received seven daily amphetamine injections. Animals were analyzed at varying times after the last injection.

Restraint stress. Rats were placed in a plastic conical sleeve (DecapiCone; model DC200; Braintree Scientific, Braintree, MA) for up to 60 min. Rats were removed from the cone and placed back into their home cage for time points exceeding $60 \mathrm{~min}$.

Construction of viral vectors. Dr. Tsonwin Hai (Ohio State University) generously provided the ATF3 cDNA. ATF2 and ATF4 were amplified from cDNA generated from rat brain mRNA and inserted into the monocistronic herpes simplex virus (HSV) PrPUC amplicon (Neve and Geller, 1995) or a newer bicistronic HSV amplicon coexpressing green fluorescent protein (GFP) from a cytomegalovirus promoter in a second transcriptional unit (Clark et al., 2002). The viral IE4/5 promoter drove HSV- mediated ATF2, ATF3, or ATF4 transcription in both vectors. All amplicons were packaged using previously described methods (Neve and Geller, 1995). A $\beta$-galactosidase (LacZ) or enhanced GFP (eGFP) viral vector was used as a control.

Stereotaxic surgery. HSV vectors were injected bilaterally ( $1 \mu \mathrm{l} /$ side in $10 \mathrm{~min}$ ) into the nucleus accumbens shell using coordinates from Paxinos and Watson (1997) (anteroposterior, 1.7; lateral, 2.4; dorsal, -6.7 $\mathrm{mm}$ from bregma; $10^{\circ}$ lateral angle) according to published procedures (Green et al., 2006). Behavioral measures were taken $48-96 \mathrm{~h}$ after surgery when HSV-mediated expression is maximal (Barrot et al., 2002). Accuracy of all stereotaxic injections was determined after the behavioral experiments, and animals with injection sites outside the nucleus accumbens shell ( $<5 \%$ of all animals studied) were not used for analysis. Some rats were subjected to multiple behavioral experiments within the 48-96 $\mathrm{h}$ time window according to a standard algorithm where anxiety and appetitive tests preceded stress or drug tests. Stress and amphetamine were not examined in the same animals.

Validation of virus. Bicistronic HSV vectors were injected into rat striatum according to previous methods (Carlezon et al., 1998; Green et al., 2006). Rats were perfused with $4 \%$ paraformaldehyde $24 \mathrm{~h}$ later. Brains were postfixed overnight and cryoprotected with $20 \%$ glycerol. Brains were sliced at $35 \mu \mathrm{m}$ for immunohistochemistry. A double fluorescent immunohistochemistry procedure was used to detect GFP and the overexpressed ATF target protein. The primary GFP antibody was from chicken (catalog \#GFP1020; 1:8000 dilution; Aves Labs, Tigard OR), and the primary ATF2, ATF3, and ATF4 antibodies were from rabbit (s.c.187, s.c.-188, and s.c.-200; 1:1000; Santa Cruz Biotechnology, Santa Cruz, CA). Secondary CY2-conjugated donkey anti-chicken and CY3conjugated donkey anti-rabbit were used at a 1:200 concentration. PC12 cells were infected with HSVs, and Western blots were used to verify size of the expressed protein.

Locomotor activity. Activity was measured in a circular corridor $(10 \mathrm{~cm}$ wide, $60 \mathrm{~cm}$ in diameter, and $30 \mathrm{~cm}$ high; Med-Associates, St. Albans, VT) via four photoelectric cells located every $90^{\circ}$ along the circle as described previously (Rahman et al., 2003). For amphetaminestimulated locomotor activity, rats were given two habituation sessions (60 min each day, beginning $48 \mathrm{~h}$ after surgery) before testing. On the amphetamine challenge day (day 4 after surgery), rats were placed in corridors for $60 \mathrm{~min}$ before being injected with $4 \mathrm{mg} / \mathrm{kg}$ (i.p.) amphetamine hemi-sulfate. Locomotor activity was assessed for an additional 120 min.

Sucrose preference and neophobia. Published procedures were used (Green et al., 2006). For sucrose preference, water was removed, and rats were pre-exposed to a $1 \%(\mathrm{w} / \mathrm{v})$ sucrose solution for $3 \mathrm{~d}$ ending at least $48 \mathrm{~h}$ before surgery. For testing, rats were first isolated at 5:00 P.M. with food but no water. When the lights went out at 7:00 P.M., preweighed water and $1 \%$ sucrose bottles were placed on the home cage, and rats were allowed to drink for $10 \mathrm{~min}$. For sucrose neophobia, rats were not preexposed to sucrose before surgery, and the duration of the test session was $30 \mathrm{~min}$.

Forced swim test. The forced swim test was performed according to published procedures (Eisch et al., 2003). Rats were placed in $24^{\circ}$ water in tubs $(60 \mathrm{~cm}$ tall filled to $45 \mathrm{~cm}$ ) for $15 \mathrm{~min}$ for the initial session and $5 \mathrm{~min}$ for the test session. The latency to immobility is defined as no struggling for a full $1 \mathrm{~s}$ and has been validated as an antidepressant-sensitive measure (Pliakas et al., 2001; Eisch et al., 2003).

Elevated plus maze. Anxiety-like behavior in the elevated plus maze was measured according to published procedures (Barrot et al., 2002). The arms of the elevated plus maze measured $12 \times 50 \mathrm{~cm}$, and the maze was $1 \mathrm{~m}$ from the floor. Time on open arms was measured for $5 \mathrm{~min}$ (expressed as a percentage of total time). Behavior was scored using an automated video-tracking system (Noldus Ethovision; Noldus Information Technology, Sterling, VA). For ATF2, the test was performed under low-light conditions. After finding no significant differences using the ATF3 and ATF4 vectors under these conditions, a different group of animals was tested under bright-light conditions, optimized for detecting anxiolytic-like effects. 

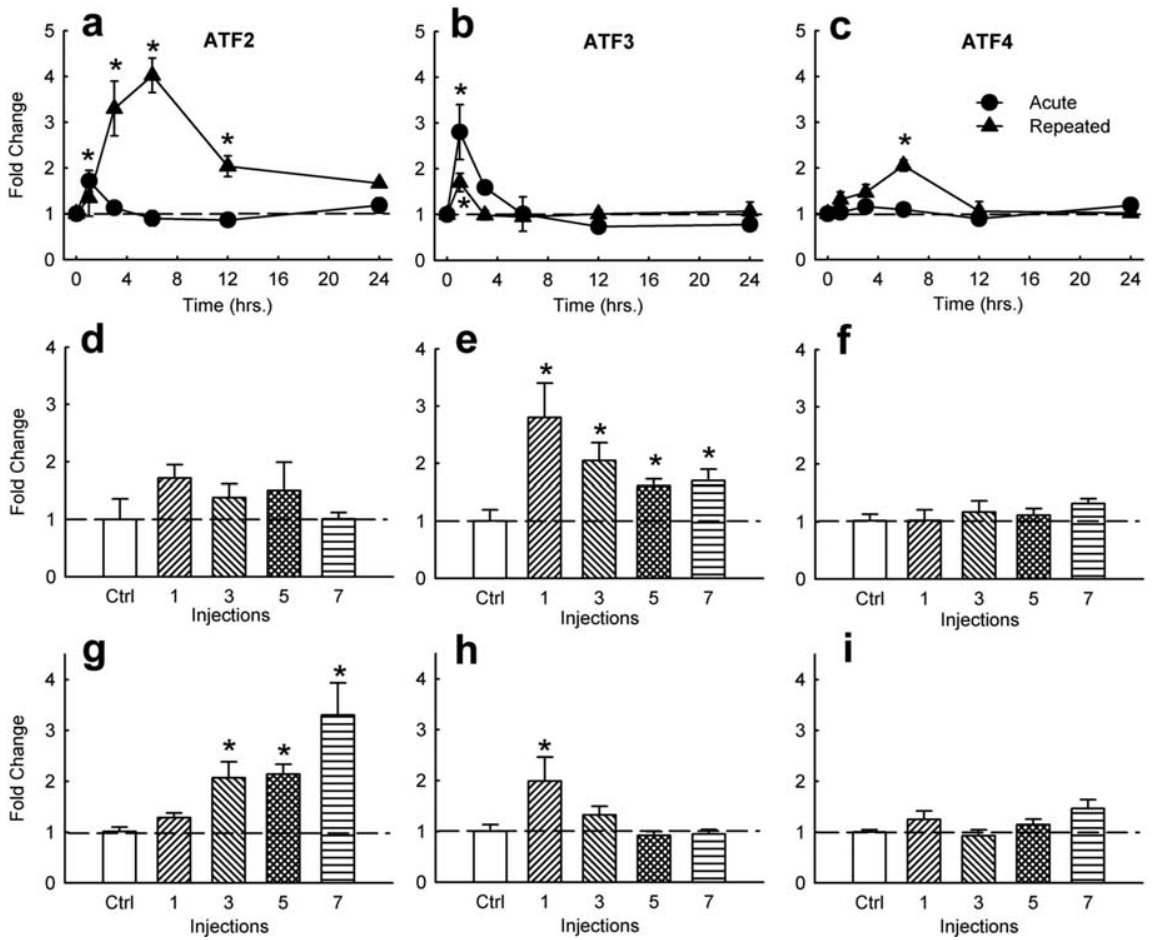

Figure 1. Effect of amphetamine on ATF2, ATF3, and ATF4 mRNA expression in striatum. Time course of acute and repeated (7 d) amphetamine-induced (4 mg/kg, i.p.) ATF2 (a), ATF3 (b), and ATF4 (c) mRNA levels. Data points represent mean \pm SEM relative induction of mRNA compared with saline-injected controls $(\mathrm{Ctrl})(n=4)$. Asterisks denote statistical difference from saline. Effect of acute and repeated amphetamine injections on ATF2 $(\boldsymbol{d}, \boldsymbol{g})$, ATF3 $(\boldsymbol{e}, \boldsymbol{h})$, and ATF4 $(\boldsymbol{f}, \boldsymbol{i})$ mRNA levels at $1 \mathrm{~h}(\boldsymbol{d}-\boldsymbol{f})$ or $3 \mathrm{~h}(\boldsymbol{g}-\boldsymbol{i})$ after the last amphetamine injection.

\section{Results}

Effect of amphetamine on ATF2, ATF3, and ATF4 expression

As a first step in our study, we examined whether amphetamine induces ATF mRNA expression in whole striatum, including nucleus accumbens. We chose amphetamine $(4 \mathrm{mg} / \mathrm{kg})$, because it has been shown previously to dramatically induce CRE-mediated transcription within this brain region (Shaw-Lutchman et al., 2003), along with increasing levels of phosphoCREB (Konradi et al., 1994) and ICER mRNA (Green et al., 2006). ATF2, ATF3, and ATF4 mRNA levels were measured by real-time PCR. A twofactor ANOVA (time $\times$ drug) of ATF2, ATF3, and ATF4 data produced a main effect of time, a main effect of drug, and a significant interaction between the two: ATF2, time, $F_{(5,63)}=6.4$, $p<0.001$, drug, $F_{(2,63)}=32.1, p<0.001$, interaction, $F_{(10,63)}=$ $10.0, p<0.001$; ATF3, time, $F_{(5,60)}=8.7, p<0.001$, drug, $F_{(2,60)}$ $=5.4, p<0.01$, interaction, $F_{(10,60)}=5.4, p<0.01$; ATF4, time, $F_{(5,61)}=4.7, p<0.01$, drug, $F_{(2,61)}=22.9, p<0.001$, and interaction, $F_{(10,61)}=2.6, p<0.05$. Acute amphetamine administration increased expression of ATF2 (Fig. 1a) and ATF3 (Fig. 1b), but not ATF4 (Fig. 1c), in the whole striatum. A subsequent experiment investigated $8 \mathrm{mg} / \mathrm{kg}$ amphetamine and still found no significant induction of ATF4 $\left(F_{(1,8)}=2.7 ; p=0.15\right)$. However, repeated amphetamine administration significantly increased mRNA levels for all three ATFs: ATF2 (Fig. 1a), ATF3 (Fig. 1b), and ATF4 (Fig. 1c). In contrast to these ATFs, we found no significant changes in levels of ATF1 or CREM $\tau$ (a major product of the crem gene) expression in response to acute or repeated amphetamine exposures (data not shown).

The three ATF mRNAs showed different time courses of induction in response to amphetamine treatment. At $1 \mathrm{~h}$ postinjection, amphetamine increased expression of ATF3 mRNA $\left(F_{(4,18)}\right.$
$=3.42 ; p<0.05)($ Fig. $1 e)$ but not ATF2 mRNA $\left(F_{(4,20)}=0.797\right.$; n.s.) (Fig. $\left.1 d\right)$ or ATF4 mRNA $\left(F_{(4,19)}=0.695\right.$; n.s.) (Fig. $1 f)$. At the $3 \mathrm{~h}$ time point, amphetamine induced expression of ATF3 mRNA $\left(F_{(4,19)}\right.$ $=4.39 ; p<0.05)($ Fig. $1 h)$ but not ATF2 or ATF4 mRNA $\left(F_{(4,19)}=0.984\right.$; n.s.) (Fig. $1 g, i)$. The three ATF mRNAs also showed different responses to repeated amphetamine treatment. Although induction of ATF3 expression waned with repeated amphetamine exposure (Fig. 1b,e,h), the induction of both ATF2 (Fig. $1 a, g$ ) and ATF4 (Fig. 1c) expression increased with chronic exposure to the drug.

To provide greater information regarding the regional pattern of ATF induction by amphetamine, similar experiments examined the effects of amphetamine at key time points on ATF mRNA expression levels within the nucleus accumbens specifically. Results of these experiments were analogous to those with whole striatum and documented the ability of chronic, but not acute, amphetamine to induce expression of ATF2 at the $3 \mathrm{~h}$ time point $\left(F_{(1,12)}=\right.$ $4.7 ; p<0.05)$, and the greater ability of acute versus chronic amphetamine to induce ATF3 expression at the $1 \mathrm{~h}$ time point $\left(F_{(1,8)}=9.1 ; p<0.05\right)$ in this brain region (Table 1). Interestingly, however, ATF4 was induced in the nucleus accumbens by both acute and chronic amphetamine at the $3 \mathrm{~h}$ time point $\left(F_{(1,12)}\right.$ $=4.3 ; p<0.05)$, whereas acute amphetamine had no effect in whole striatum.

\section{Effect of stress on ATF2, ATF3, and ATF4 expression}

To study the effect of aversive environmental stimuli on ATF expression in nucleus accumbens and dorsal striatum, we subjected rats to acute restraint stress and analyzed the animals after varying times of stress. This stress treatment has been shown to alter levels of CRE activity, phosphoCREB, and ICER mRNA levels in these brain regions (Pliakas et al., 2001; Barrot et al., 2002; Green et al., 2006). ANOVAs of these time course data showed that acute restraint stress produced a transient decrease in levels of ATF2 mRNA in whole striatum followed by a small increase at longer time points $\left(F_{(5,23)}=9.39 ; p<0.001\right)$ (Fig. $\left.2 a\right)$. In contrast, acute restraint stress caused a dramatic $(\sim 20$-fold $)$ increase in ATF3 mRNA levels, which peaked after 30 min of stress $\left(F_{(5,23)}=30.93 ; p<0.001\right)$ (Fig. $\left.2 b\right)$. Acute restraint stress did not significantly alter levels of ATF4 mRNA in this brain region (Fig. 2c). The increase in ATF2 mRNA with repeated stress also did not reach statistical significance (Fig. $2 d$ ). However, repeated stress significantly induced ATF4 mRNA $\left(F_{(4,18)}=5.82\right.$; $p<0.01)$, and the induction of ATF3 waned with repeated stress $\left(F_{(4,18)}=33.92 ; p<0.001\right)$ (Fig. $2 e$ ), similar to the shifting patterns seen for both ATFs after chronic amphetamine.

\section{Validation of HSV vectors}

To study the functional role played by ATF2, ATF3, and ATF4 in nucleus accumbens in responses to amphetamine and stress, we engineered HSV vectors to overexpress each of these proteins. Examples of the validation of these new dual-cistronic vectors is 
shown in Fig. 3. Immunohistochemistry demonstrates coexpression of the GFP reporter protein (Fig. 3a) along with ATF3 (Fig. $3 b$ ). Coexpression of GFP and target protein was $>95 \%$ for each of the vectors. Additionally, overexpressed protein from PC12 lysates was analyzed via Western blot to verify size of the expressed protein (data not shown). As seen with other HSV vectors (Carlezon et al., 1998; Barrot et al., 2002; Green et al., 2006), transgene expression was maximal 2-4 d after virus injection and was not associated with toxicity in excess of that seen after vehicle injections alone (data not shown). Additionally, none of the viruses had an effect on body weight $\left(\mathrm{ATF} 2, F_{(1,16)}=0.38, p=0.55\right.$; ATF3, $F_{(1,16)}=0.07, p=0.79 ; \mathrm{ATF} 4, F_{(1,16)}=$ $0.013 ; p=0.91)$.

\section{Effect of ATF2, ATF3, or ATF4} overexpression on locomotor activity Using viral-mediated gene transfer in the shell of the nucleus accumbens, we found that ATF2 overexpression increased sensitivity to amphetamine-stimulated locomotor activity $\left(F_{(1,11)}=3.25 ; p<0.05\right.$, one tailed test based on the established effects of ICER on locomotor activity) (Green et al., 2006) (Fig. 4d). In contrast, ATF3 overexpression produced a robust decrease in amphetamine-stimulated locomotor activity $\left(F_{(1,14)}=15.18 ; p<0.005\right)$ (Fig. $4 e$ ). ATF2 and ATF3 had no effect on spontaneous locomotor activity $\left(\mathrm{ATF} 2, F_{(1,14)}=\right.$ 0.93, $p=0.35$; ATF3, $F_{(1,14)}=0.01, p=$ 0.92); however, ATF4 slightly increased spontaneous activity compared with control but only during the first habituation session $\left(F_{(1,14)}=5.4 ; p<0.05\right)$. None of the ATFs affected locomotor activity after saline injection [ATF2, $F_{(1,14)}=0.54, p=$ 0.47 ; ATF3, $F_{(1,14)}=1.9, p=0.19 ; \mathrm{ATF} 4$, $F_{(1,14)}=0.04, p=0.85$ (Fig. $4 a-c$, respectively)].

\section{Effect of ATF2, ATF3, or ATF4}

\section{overexpression on}

\section{anxiety-related behavior}

To further understand the functional relevance of ATF2, ATF3, and ATF4 in the nucleus accumbens, rats overexpressing each of these transcription factors, by use of HSV vectors, were tested in the elevated plus maze. Overexpression of ATF2 caused an increase in anxiety-like behavior: decreased time spent on the open arm during the low-light condition $\left(F_{(1,12)}=\right.$ 5.32; $p<0.05$ ) (Fig. 5a). In contrast, overexpression of ATF3 $\left(F_{(1,10)}=5.42 ; p<0.05\right)$ (Fig. $\left.5 b\right)$ or $\operatorname{ATF} 4\left(F_{(1,10)}=5.55 ; p<\right.$ 0.05 ) (Fig. $5 c$ ) caused an anxiolytic-like response: increased time on the open arm only under anxiogenic conditions. Neither ATF2 nor ATF4 altered total distance moved on the elevated plus maze $\left[\mathrm{ATF} 2, F_{(1,12)}=0.01, p=0.91\right.$ (Fig. $5 d$ ); ATF4, $F_{(1,10)}=$ 0.33, $p=0.58$ (Fig. 5f)]; however, ATF3 expression decreased
Table 1. Effect of amphetamine on ATF2, ATF3, and ATF4 mRNA expression in the nucleus accumbens

\begin{tabular}{llll}
\hline Conditions & ATF2 (3 h) & ATF3 (1 h) & ATF4 (3 h) \\
\hline Acute amph & & & \\
$\quad$ Sal & $1.00 \pm 0.03$ & $1.00 \pm 0.09$ & $1.00 \pm 0.09$ \\
$\quad$ Amph & $0.98 \pm 0.05$ & $1.87 \pm 0.26^{*}$ & $1.79 \pm 0.30^{*}$ \\
Repeated amph & & & \\
$\quad$ Sal & $1.00 \pm 0.03$ & $1.00 \pm 0.09$ & $1.00 \pm 0.09$ \\
Amph & $1.12 \pm 0.03^{*}$ & $1.12 \pm 0.12$ & $1.49 \pm 0.08^{*}$ \\
\hline
\end{tabular}

Values are mean \pm SEM fold induction of mRNA. Asterisks denote statistical difference from saline control group (Sal). amph, Amphetamine group; Acute, single injection; Repeated, 7 daily injections. 

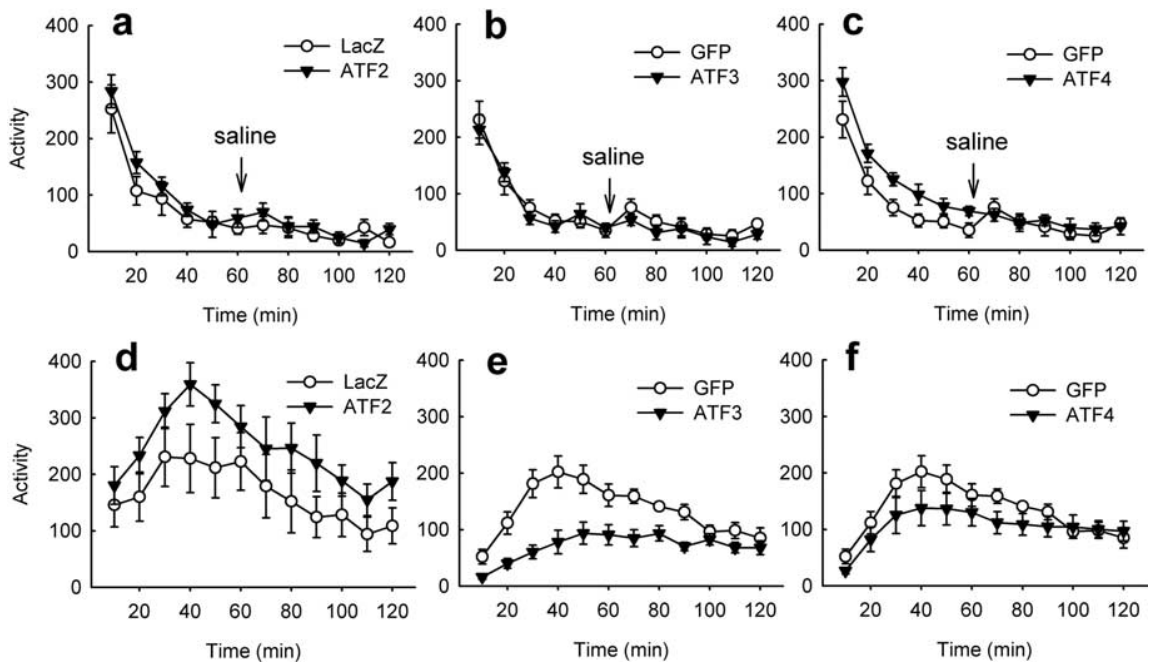

Figure 4. Effect of viral-mediated overexpression of ATF2, ATF3, or ATF4 in nucleus accumbens on spontaneous and amphetamine-stimulated locomotor activity. Time course of spontaneous locomotor activity for HSV-ATF2 (a), -ATF3 (b), and -ATF4 (c) and saline-induced activity. Bottom panels show amphetamine-stimulated locomotor activity ( $2 \mathrm{mg} / \mathrm{kg}$, i.p.) $3 \mathrm{~d}$ after bilateral intra-NAc injections of HSV-ATF2 $(\boldsymbol{d})$, -ATF3 $(\boldsymbol{e})$, or -ATF4 $(\boldsymbol{f})$ when transgene expression is maximal. Control animals received injections of HSV-GFP. Points represent mean \pm SEM beam breaks in $10 \mathrm{~min}$ bins $(n=6-8)$. Rats were allowed 60 min to habituate to the test chamber before amphetamine was injected.
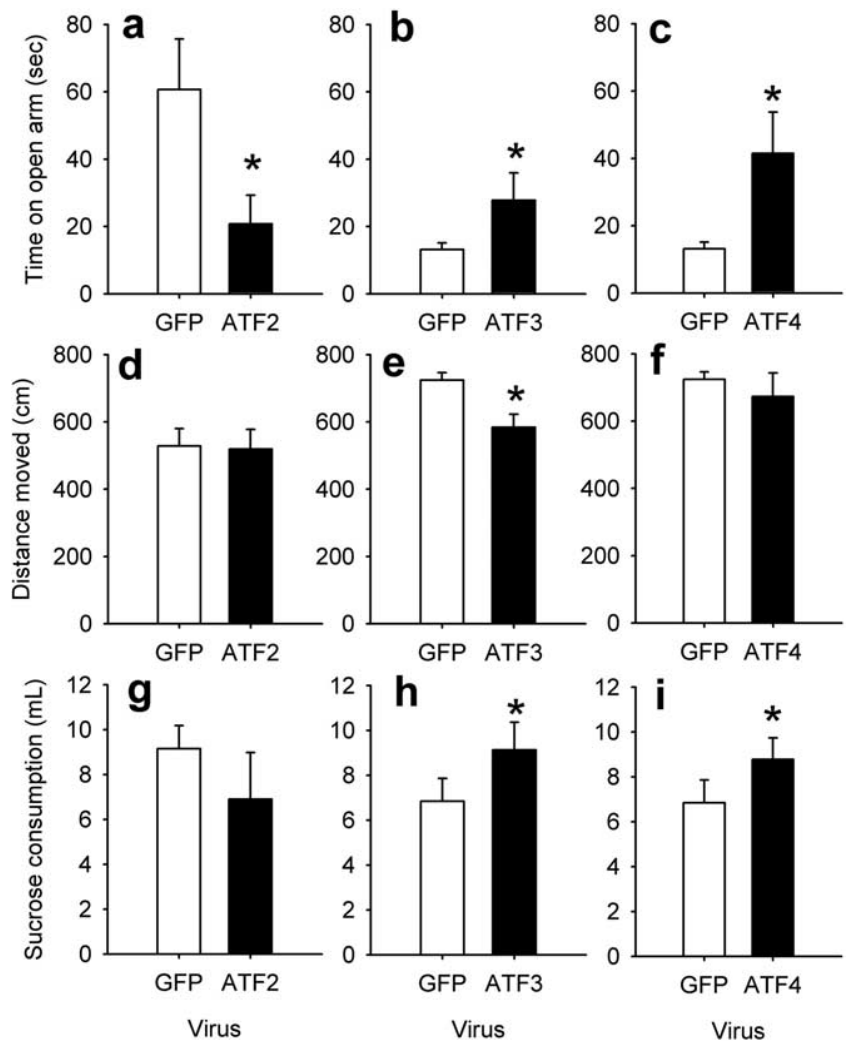

Figure 5. Effect of viral-mediated overexpression of ATF2, ATF3, or ATF4 in the nucleus accumbens on anxiety-related behavior. HSV vectors overexpressing GFP (control), ATF2, ATF3, or ATF4 were injected bilaterally in the nucleus accumbens. $\boldsymbol{a}-\boldsymbol{c}$, Effect of ATF2, ATF3, or ATF4 on time spent in the open arm of the elevated plus maze. Error bars represent mean \pm SEM time spent on the open arms during a 5 min session. The ATF2 experiment was conducted under a low-anxiety condition, and the ATF3 and ATF4 experiments were conducted under high-anxiety (brighter light) conditions $(n=4-8) . \boldsymbol{d}-\boldsymbol{f}$, Total distance moved on the elevated plus maze for rats overexpressing ATF2, ATF3, or ATF4. $\boldsymbol{g}-\boldsymbol{i}$, Sucrose neophobia subsequent to overexpression of ATF2, ATF3, or ATF4. Error bars represent milliliter of a $1 \%$ sucrose solution consumed by naive rats across a 30 min session $(n=4-8)$. Asterisks denote significant difference from HSV-GFP control. total distance on the elevated plus maze $\left(F_{(1,10)}=7.3 ; p<0.05\right)$ (Fig. $5 e$ ).

To further investigate anxiety-like behavior, rats were tested for sucrose neophobia. Consistent with the elevated plus maze data, $\operatorname{ATF} 3\left(F_{(1,15)}=5.57 ; p<0.05\right)$ (Fig. 5h) and ATF4 $\left(F_{(1,14)}=7.38 ; p<\right.$ $0.05)$ (Fig. 5i) increased sucrose consumption, whereas ATF2 had no effect (Fig. $5 g$ ).

\section{Effect of ATF2, ATF3, or ATF4 overexpression on depression-related behavior}

The forced swim test is one of the most widely used assays for depression- and antidepressant-like behavior (Cryan et al., 2005). HSV-mediated overexpression of ATF2 produced an antidepressant effect in the forced swim test by increasing latency to immobility $\left(F_{(1,12)}=5.62 ; p<0.05\right)$ (Fig. $6 a$ ) and decreasing total duration of immobility $\left(F_{(1,12)}=15.28 ; p<0.01\right)$ (Fig. $6 d)$. In contrast, the opposite results were observed with overexpression of ATF3 $\left(F_{(1,14)}=8.58 ; p<0.05\right)($ Fig. $6 b)$ or ATF4 $\left(F_{(1,14)}=10.13 ; p<0.01\right)$ (Fig. $\left.6 c\right)$, consistent with an increase in depression-like behavior with these two transcription factors. ATF3 and ATF4 also increased total duration of immobility $\left[\mathrm{ATF} 3, F_{(1,14)}=10.1, p<0.01\right.$ (Fig. 6e); ATF4, $F_{(1,14)}=14.29$, $p<0.01$ (Fig. $6 f$ )]. One hallmark of depression is reduced sensitivity to rewarding stimuli. Accordingly, we tested preference for sucrose, a natural reward, in animals with sucrose-drinking experience to avoid neophobic responses (Fig. 6g-i). HSVmediated overexpression of each of the ATFs caused distinctive responses. ATF2 overexpression significantly decreased water intake $\left(F_{(1,16)}=4.78 ; p<0.05\right)$ (Fig. $6 g$ ) and increased sucrose intake $\left(F_{(1,16)}=4.85 ; p<0.05\right)$. ATF3 overexpression increased water consumption but not sucrose drinking $\left(F_{(1,13)}=2.99 ; p=\right.$ 0.11 ) (Fig. 6h). ATF4 overexpression decreased sucrose consumption with no change in water intake $\left(F_{(1,12)}=4.66 ; p<\right.$ 0.05) (Fig. 6i).

\section{Discussion}

Results of the present study provide novel evidence that ATF2, ATF3, and ATF4 in the nucleus accumbens function to regulate an individual's responses to emotional stimuli. We show that the mRNAs for each of these transcription factors are induced by amphetamine and by restraint stress in striatum, and that increased expression of these proteins specifically in the nucleus accumbens (part of the ventral striatum), achieved via viralmediated gene transfer, alters behavioral responses to amphetamine and stress. The three ATFs exhibit distinct patterns of induction by amphetamine and stress. Although ATF2 and ATF4 induction increases with repeated drug and stress exposures, ATF3 induction resembles that of several immediate early genes, such as c-Fos, with rapid, transient induction seen with acute amphetamine and stress and diminishing induction seen with repeated exposure. In contrast to ATF2-4, we failed to observe amphetamine or stress regulation of ATF1 or CREM $\tau$ expression in this brain region.

Our behavioral experiments offer strong evidence that ATF2, ATF3, and ATF4 each modulate complex behavior when induced in the nucleus accumbens. The results show that viral-mediated 
overexpression of ATF2 in this brain region increases behavioral responsiveness to salient environmental stimuli, an effect similar to that seen with overexpression in the nucleus accumbens of either ICER or mCREB, both dominant-negative antagonists of CREB (Carlezon et al., 1998; Barrot et al., 2002; Newton et al., 2002; Green et al., 2006). The ATF2 phenotype is, therefore, equivalent to that seen after inhibition of CREB activity. ATF2 increased locomotor responses to amphetamine administration compared with rats expressing only GFP as a control. Additionally, ATF2 increased anxiety-like behavior, based on decreased time spent on the open arm of an elevated plus maze and on increased neophobia to a novel sucrose taste. ATF2 expression also decreased depression-like behavior (that is, it decreased immobility) in the forced swim test and increased consumption of a familiar sucrose solution.

In contrast, viral-mediated overexpression of ATF3 or of ATF4 produced opposite behavioral responses, compared with ATF2, in each of these experimental paradigms. The behavioral phenotype induced by these transcription factors, therefore, is equivalent to that seen with CREB (Carlezon et al., 1998; Barrot et al., 2002). ATF3 and ATF4 each decreased amphetaminestimulated locomotor activity, decreased measures of anxiety-like behavior in the elevated plus maze and sucrose neophobia tests, and increased measurements of depression-like behavior, based on increased immobility in the forced swim test and reduced preference for a familiar sucrose solution. When combined with previous findings from our laboratory (Carlezon et al., 1998; Pliakas et al., 2001; Barrot et al., 2002; Sakai et al., 2002; Green et al., 2006), these data show that induction of CREB/ATF family transcription factors in the nucleus accumbens produces one of two highly consistent behavioral phenotypes (Table 2): decreased responsiveness to emotional stimuli (CREB, ATF3, and ATF4) or, the opposite, increased emotional reactivity (ICER and ATF2).

It is interesting to note that regulation of ATF proteins causes opposite modulation of anxiety- and depression-related behavior in rats, whereas anxiety and depression are often linked in humans. It should be noted, however, that many instances of depression are not associated with anxiety symptoms, and that CREB-family proteins may be involved in this subset of patients. Moreover, several CREB-family proteins studied thus far in the nucleus accumbens (Table 2) modulate anxiety- and depressionrelated behavior in opposite directions.

The transcriptional activity of ATF2, ATF3, and ATF4 has remained poorly characterized, particularly in brain. There are reports that each of these proteins can function as a transcriptional activator or repressor in various experimental systems (Hai and Hartman, 2001; Nakagomi et al., 2003). Moreover, the degree to which the proteins regulate transcription via CRE sites is
Table 2. Effects of CREB/ATF family transcription factors in the nucleus accumbens on emotional behavior

\begin{tabular}{llll}
\hline CREB family protein & Anxiety-like behavior & Depression-like behavior & Drug sensitivity \\
\hline CREB & $\downarrow$ & $\uparrow \uparrow$ & $\downarrow \downarrow$ \\
ATF3 & $\downarrow \downarrow$ & $\uparrow$ & $\downarrow \downarrow$ \\
ATF4 & $\downarrow \downarrow$ & $\uparrow \uparrow$ & $\downarrow$ \\
ICER & $\uparrow \uparrow$ & $\downarrow \downarrow \downarrow$ & $\uparrow \uparrow$ \\
ATF2 & $\uparrow$ & $\downarrow \downarrow$ & $\uparrow$ \\
\hline
\end{tabular}

The table summarizes the behavioral effects of overexpression of each protein in the nucleus accumbens based on data, in addition to the present study, reported by Carlezon et al. (1998), Pliakas et al. (2001), Barrot et al. (2002), and Green et al. (2006). The ICER/ATF2 phenotype represents a"CREB antagonist" phenotype, because overexpression of mCREB, a dominant-negative CREB mutant, produces equivalent effects.

unknown. Thus, in addition to inconsistent effects on CREmediated transcription, ATF2, ATF3, and ATF4 can act on CREB-insensitive CRE-like sites (Benbrook and Jones, 1994). The three ATFs can also dimerize with c-Jun or other bZIP transcription factors and bind to AP-1 or to CRE-AP-1 hybrid sites (Hai and Curran, 1991; De Cesare et al., 1995). Our findings clearly establish that, within the nucleus accumbens in vivo, ATF2 
functions directly or indirectly as an antagonist of CRE-mediated transcription, whereas ATF3 and ATF4 function as activators of CRE-mediated transcription. These observations thereby provide novel insight to the functional activity of ATF2, ATF3, and ATF4 in the brain in vivo.

Psychological stress has been linked to cellular stress and DNA damage (for review, see Gidron et al., 2006). It is, therefore, interesting to note that restraint stress caused a nearly 20 -fold increase in ATF3 mRNA levels in striatal regions, which is fivefold greater than the induction seen with a relatively high dose of amphetamine. This observation raises the possibility that stress induction of ATF3 may be mediated via activation of intracellular stress cascades as opposed to being mediated by increased dopaminergic transmission. Thus, although very high doses of methamphetamine can cause cell death via cellular stress pathways (Hebert and O'Callaghan, 2000; Jayanthi et al., 2004), there is no evidence that the amphetamine dose used in the present experiments activates cellular stress pathways. In contrast, ATF3 is known to be induced in non-nervous tissue by nonstress-related stimuli (Cho et al., 2001). Consequently, additional work is needed to better understand the cellular pathways through which amphetamine and stress exposures induce ATF3 in striatum.

An interesting question raised by the present studies is whether members of the CREB/ATF family regulate transcription by altering the levels of expression of other family members. There is no precedent for any of the ATFs regulating CREB expression in brain. Rather, CREB is known to induce ICER and other repressors from the crem gene, which then feed back, with some delay, to inhibit CREB-mediated transcription. There is also a precedent of ATF4 regulating gene transcription via induction of ATF3 by directly binding to and activating the atf 3 gene promoter (Jiang et al., 2004). Thus, it is conceivable that some of the effects seen for ATF4 overexpression may be mediated via ATF3. However, our data do not support this possibility, because we see progressively enhanced induction of ATF4 expression in striatum after chronic amphetamine or stress exposures, conditions where induction of ATF3 becomes desensitized. It will be interesting in future studies to determine whether CREB, ICER, and the various ATFs regulate emotional behavior, at the level of the nucleus accumbens, via the regulation of a set of common target genes.

We show that amphetamine increases ATF2 expression in striatum, and that ATF2 increases locomotor responses to amphetamine. Although hypothetical at this point, these findings raise the possibility that ATF2 induction may contribute to mechanisms for behavioral sensitization to amphetamine. Likewise, induction of ATF2 may contribute to sensitization to repeated stress exposures as well as to cross-sensitization between stress and stimulants (Covington and Miczek, 2001). In contrast, amphetamine increases ATF3 and ATF4 expression, which decrease behavioral responsiveness to amphetamine, suggesting that these proteins may contribute to tolerance to some amphetamine-induced behaviors (Kuo and Cheng, 2002). What remains unknown, however, is how sustained overexpression of an ATF (i.e., via viral vectors) may be different from phasic, transient changes in expression induced by amphetamine or stress. Also requiring additional study is whether dimerization with other bZip transcription factors (e.g., c-Jun) may be different for viral versus endogenous ATF expression. Regardless, these experiments demonstrate that ATF transcription factors are capable of altering a variety of emotional behaviors when overexpressed in nucleus accumbens.

Together, findings of the present study offer novel informa- tion about the functions of ATF2, ATF3, and ATF4 in the nucleus accumbens. The expression of all three transcription factors is induced in this brain region by amphetamine or stress, and then regulate the responsiveness of the individual to these behavioral stimuli. The findings, therefore, extend the role played by CREB/ ATF family transcription factors in the reward circuits of the brain in the regulation of emotional behavior well beyond CREB itself, and demonstrate that several family members mediate distinct behavioral phenotypes at the level of this brain circuit.

\section{References}

Barrot M, Olivier JD, Perrotti LI, DiLeone RJ, Berton O, Eisch AJ, Impey S, Storm DR, Neve RL, Yin JC, Zachariou V, Nestler EJ (2002) CREB activity in the nucleus accumbens shell controls gating of behavioral responses to emotional stimuli. Proc Natl Acad Sci USA 99:11435-11440.

Benbrook DM, Jones NC (1994) Different binding specificities and transactivation of variant CRE's by CREB complexes. Nucl Acids Res 22:1463-1469.

Blendy JA (2006) The role of CREB in depression and antidepressant treatment. Biol Psychiatry 59:1144-1150.

Carlezon Jr WA, Thome J, Olson VG, Lane-Ladd SB, Brodkin ES, Hiroi N, Duman RS, Neve RL, Nestler EJ (1998) Regulation of cocaine reward by CREB. Science 282:2272-2275.

Cho RJ, Huang M, Campbell MJ, Dong H, Steinmetz L, Sapinoso L, Hampton G, Elledge SJ, Davis RW, Lockhart DJ (2001) Transcriptional regulation and function during the human cell cycle. Nat Genet 27:48-54.

Clark MS, Sexton TJ, McClain M, Root D, Kohen R, Neumaier JF (2002) Overexpression of 5-HT1b receptor in dorsal raphe nucleus using herpes simplex virus gene transfer increases anxiety behavior after inescapable stress. J Neurosci 22:4550-4562.

Covington HE, Miczek KA (2001) Repeated social-defeat stress, cocaine or morphine. Effects on behavioral sensitization and intravenous cocaine self-administration "binges." Psychopharmacology 158:388-398.

Cryan JF, Valentino RJ, Lucki I (2005) Assessing substrates underlying the behavioral effects of antidepressants using the modified rat forced swimming test. Neurosci Biobehav Rev 29:547-569.

De Cesare D, Vallone D, Caracciolo A, Sassone-Corsi P, Nerlov C, Verde P (1995) Heterodimerization of c-Jun with ATF-2 and c-Fos is required for positive and negative regulation of the human urokinase enhancer. Oncogene 11:365-376.

Eisch AJ, Bolanos CA, de Wit J, Simonak RD, Pudiak CM, Barrot M, Verhaagen J, Nestler EJ (2003) Brain-derived neurotrophic factor in the ventral midbrain-nucleus accumbens pathway: a role in depression. Biol Psychiatry 54:994-1005.

Gidron Y, Russ K, Tissarchondou H, Warner J (2006) The relation between psychological factors and DNA-damage: a critical review. Biol Psychol 72:291-304.

Green TA, Alibhai IN, Hommel JD, DiLeone RJ, Kumar A, Theobald DE, Neve RL, Nestler EJ (2006) Induction of inducible cAMP early repressor (ICER) in nucleus accumbens by stress or amphetamine increases behavioral responses to emotional stimuli. J Neurosci 26:8235-8242.

Hai T, Curran T (1991) Cross-family dimerization of transcription factors Fos/Jun and ATF/CREB alters DNA binding specificity. Proc Natl Acad Sci USA 88:3720-3724.

Hai T, Hartman MG (2001) The molecular biology and nomenclature of the activating transcription factor/cAMP responsive element binding family of transcription factors: activating transcription factor proteins and homeostasis. Gene 273:1-11.

Hai T, Wolfgang CD, Marsee DK, Allen AE, Sivaprasad U (1999) ATF3 and stress responses. Gene Expr 7:321-335.

Hebert MA, O'Callaghan JP (2000) Protein phosphorylation cascades associated with methamphetamine-induced glial activation. Ann NY Acad Sci 914:238-262.

Jayanthi S, Deng X, Noailles PA, Ladenheim B, Cadet JL (2004) Methamphetamine induces neuronal apoptosis via cross-talks between endoplasmic reticulum and mitochondria-dependent death cascades. FASEB J 18:238-251.

Jiang HY, Wek SA, McGrath BC, Lu D, Hai T, Harding HP, Wang X, Ron D, Cavener DR, Wek RC (2004) Activating transcription factor 3 is integral to the eukaryotic initiation factor 2 kinase stress response. Mol Cell Biol 24:1365-1377. 
Konradi C, Cole RL, Heckers S, Hyman SE (1994) Amphetamine regulates gene expression in rat striatum via transcription factor CREB. J Neurosci 14:5623-5634.

Kuo DY, Cheng JT (2002) Role of cerebral dopamine but not plasma insulin, leptin and glucocorticoid in the development of tolerance to the anorectic effect of amphetamine. Neurosci Res 44:63-69.

Laifenfeld D, Karry R, Grauer E, Klein E, Ben-Shachar D (2004) ATF2, a member of the CREB/ATF family of transcription factors, in chronic stress and consequent to antidepressant treatment: animal models and human post-mortem brains. Neuropsychopharmacology 29:589-597.

Lu D, Chen J, Hai, T (2007) The regulation of ATF3 gene expression by mitogen-activated protein kinases. Biochem J 401:559-567.

Nakagomi S, Suzuki Y, Namikawa K, Kiryu-Seo S, Kiyama H (2003) Expression of the activating transcription factor 3 prevents c-Jun N-terminal kinase-induced neuronal death by promoting heat-shock protein $27 \mathrm{ex}$ pression and AKT activation. J Neurosci 23:5187-5196.

Neve RL, Geller AI (1995) A defective herpes simplex virus vector system for gene delivery into the brain: comparison with alternative gene delivery systems and usefulness for gene therapy. Clin Neurosci 3:262-267.

Newton SS, Thome J, Wallace T, Shirayama Y, Dow A, Schlesinger L, Duman CH, Sakai N, Chen JS, Neve R, Nestler EJ, Duman RS (2002) Inhibition of CREB or dynorphin in the nucleus accumbens produces an antidepressant-like effect. J Neurosci 22:10883-10890.

Paxinos G, Watson C (1997) The rat brain in stereotaxic coordinates. San Diego: Academic.

Pittenger C, Fasano S, Mazzocchi-Jones D, Dunnett SB, Kandel ER, Brambilla
R (2006) Impaired bidirectional synaptic plasticity and procedural memory formation in striatum-specific cAMP response element-binding protein-deficient mice. J Neurosci 26:2808-2813.

Pliakas AM, Carlson RR, Neve RL, Konradi C, Nestler EJ, Carlezon WAJ (2001) Altered responsiveness to cocaine and increased immobility in the forced swim test associated with elevated cAMP response elementbinding protein expression in nucleus accumbens. J Neurosci 21:7397-7403.

Rahman Z, Schwarz J, Zachariou V, Gold SJ, Wein M, Choi KH, Kovoor A, Chen CK, DiLeone RJ, Schwarz SC, Selley DE, Sim-Selley LJ, Barrot M, Leudtke RR, Self DW, Neve RL, Lester HA, Simon MI, Nestler EJ (2003) RGS9 modulates dopamine signaling in striatum. Neuron 38:941-952.

Sakai N, Thome J, Chen JS, Kelz MB, Steffen C, Nestler EJ, Duman RS (2002) Inducible and brain region specific CREB transgenic mice. Mol Pharmacol 61:1453-1464.

Shaw-Lutchman TZ, Impey S, Storm D, Nestler EJ (2003) Regulation of CRE-mediated transcription in mouse brain by amphetamine. Synapse 48:10-17.

Walters CL, Blendy JA (2001) Different requirements for cAMP response element binding protein in positive and negative reinforcing properties of drugs of abuse. J Neurosci 21:9438-9444.

Walters CL, Kuo YC, Blendy JA (2003) Differential distribution of CREB in the mesolimbic dopamine reward pathway. J Neurochem 87:1237-1244.

Wek RC, Jiang HY, Anthony TG (2006) Coping with stress: eIF2 kinases and translational control. Biochem Soc Trans 34:7-11. 